\title{
Produtividade Acumulada (PAC) das Matrizes em Rebanhos Nelore do Norte e Nordeste do Brasil ${ }^{1}$
}

\author{
Danielle Maria Machado Ribeiro Azevêdo², Raimundo Martins Filho ${ }^{3}$, Raimundo Nonato \\ Braga Lôbo ${ }^{4}$, Raysildo Barbosa Lôbo ${ }^{5}$, Arlindo de Alencar Araripe N. Moura ${ }^{6}$, Edgard \\ Cavalcanti Pimenta Filho7, Carlos Henrique Mendes Malhado ${ }^{8}$
}

\begin{abstract}
RESUMO - A produtividade acumulada (PAC)é um índice que indica precocidade sexual, periodicidade reprodutiva e potencial de produção de quilogramas de bezerros de matrizes bovinas. Foram analisadas 2.816 observações de fêmeas Nelore criadas em diferentes rebanhos do norte e nordeste do Brasil, recém incorporados ao Programa de Melhoramento Genético da Raça Nelore (PMGRN), com o objetivo de estimar os componentes de variância genética e residual e a herdabilidade da PAC. A média e o desvio-padrão observados para PAC foram $96,74 \pm 46,70$ $\mathrm{kg}$ de bezerros desmamados por vaca ao ano, com coeficiente de variação de 48,27\%, inferior à média obtida para o conjunto de rebanhos integrantes do PMGRN. Fêmeas da raça Nelore Padrão obtiveram melhor resultado para PAC que às fêmeas Mocha. A herdabilidade estimada foi igual a $0,11 \pm 0,06$, sugerindo a existência de variabilidade genética suficiente para a inclusão desta característica em programas de melhoramento genético, ressaltando-se, no entanto, que seu progresso depende, sobretudo, de práticas de manejo mais eficientes.
\end{abstract}

Palavras-chave: eficiência reprodutiva, fêmea zebu, herdabilidade, índice de seleção

\section{Accumulated Productivity of Nellore Cows Raised in North and Northeast of Brazil}

ABSTRACT - The accumulated productivity (ACP) is an index that takes into account the total weight of calves weaned per cow (in $\mathrm{kg}$ ), total time required for producing the calves and age at first calving. A study was conducted to estimate the components of genetic and residual variance and estimate the heritability of ACP index. Data set consisted of 2,816 observations of Nelore cows raised in different farms of the Brazilian North and Northeast. These farms participate in the Genetic Improvement Program of the Nelore Breed. The observed mean and respective standard deviation for ACP was $96.74 \pm 46.70 \mathrm{~kg}$ of weaned calves/cow/year, with a coefficient of variation of $48.27 \%$. This mean ACP, however, is lower than values estimated in other herds of the Nelore genetic program. Also, Standard Nelore cows showed higher ACP in comparison to cows of the Nelore Polled variety. Estimated heritability for ACP was $0.11 \pm 0.06$, suggesting that there is sufficient genetic variability that justify the use of ACP in genetic selection. However, results of such strategy depend, mainly, on efficient herd management.

Key Words: heritability, reproductive efficiency, selection index, zebu

\section{Introdução}

Em um mercado de grande competição como o atual, deve-se buscar a máxima produtividade para se otimizar a lucratividade. Neste contexto, em pecuária de corte, a produtividade do sistema está intimamente relacionada à eficiência reprodutiva das fêmeas que o compõem, visto que fêmeas mais precoces e férteis proporcionam aumento na taxa de nascimentos do rebanho, possibilitando maior produção de carne por ano.
Nesse sentido, a eficiência reprodutiva destacase em programas de melhoramento como critério de seleção de fêmeas (Lôbo et al., 2004), adotando-se diferentes fórmulas para a avaliação desta eficiência em vacas de corte (Dinkel \& Brown, 1978; Euclides Filho et al., 1984; Kirkpatrick et al., 1985; Rahnefeld et al., 1993). Todas essas fórmulas aliam características do bezerro e da matriz, admitindo-se que o ideal é que a vaca desmame bezerros pesados e, ao mesmo tempo, consiga manter condição corporal para parir um bezerro anualmente.

\footnotetext{
${ }^{1}$ Parte da tese de Doutorado em Zootecnia apresentada pelo primeiro autor à Universidade Federal do Ceará, realizada com financiamento do CNPq/CAPES.

2 Médica Veterinária, Doutora, Bolsista de DCR CNPq/FAPEPI no DZO/UFPI (danizootec3@hotmail.com).

${ }^{3}$ Médico Veterinário, Doutor, Bolsista de DCR CNPq/FAPEPI no DZO/UFPI (rmartinsfilho@yahoo.com.br)

${ }^{4}$ Médico Veterinário, Doutor, Pesquisador EMBRAPA Caprinos (lobo@cnpc.embrapa.br).

5 Médico Veterinário, Doutor, Professor USP (rayblobo@genbov.fmrp.usp.br).

6 Engenheiro Agrônomo, Doutor, Professor UFC (amoura@ufc.br).

7 Zootecnista, Doutor, Professor UFPB (edgard@cca.ufpb.br).

8 Médico Veterinário, Mestre, Professor UESB (chmmalhado@hotmail.com).
} 
Considerando-se que a rentabilidade do sistema em pecuária de corte está relacionada ao peso do animal ao abate, uma boa matriz deve ser capaz de aliar desempenho reprodutivo e habilidade materna. Frente a estas múltiplas exigências, a avaliação de uma fêmea no contexto produtivo necessita do estabelecimento de índices que englobem, além de características reprodutivas, sua habilidade materna, refletida em sobrevivência e maior peso das suas crias ao desmame, determinando seu valor econômico.

Notter (1995) ressalta que a herdabilidade dos índices tende a ser controlada pela característica mais variável e menos herdável. Em adição, Mercadante et al. (1996) citam que, devido à incorporação em um mesmo índice de características de herdabilidades e importâncias econômicas diferentes, a utilização de índices deve ser realizada com precaução.

Lôbo et al. (1990) propuseram uma maneira simples de avaliação da produtividade de fêmeas (Lôbo, 1994). Considerando que a comercialização dos animais em bovinocultura de corte ocorre, geralmente, após o desmame, os autores criaram um índice incluindo a fertilidade da matriz e sua habilidade materna. Este índice, denominado fertilidade real (FR=PBDx365/intervalo de partos), é expresso em quilogramas de bezerro desmamado (PBD) por ano, corrigido por um fator que relaciona o intervalo de partos ideal de 365 dias com o obtido pela matriz. Percebe-se, no entanto, que a fórmula de fertilidade real tem o inconveniente de incluir o intervalo de partos, não contemplando as fêmeas que pariram apenas uma vez ou aquelas que nunca pariram. Alguns autores (Campello et al., 1999; Gonçalves et al., 1996; Mercadante et al., 1996) trabalharam com a avaliação da fertilidade real em rebanhos da raça Nelore.

Um índice mais recente - produtividade acumulada (PAC) - tem sido estudado para avaliação da fertilidade das matrizes (Rosa, 1999; Schwengber, 2001). A PAC indica a produtividade da matriz, em quilogramas de bezerros desmamados por ano, e avalia a mesma quanto à precocidade e periodicidade reprodutiva e, ainda, quanto à sua habilidade materna, por meio de sua capacidade em desmamar crias com maior peso (Lôbo et al., 2000).

A PAC, por refletir outras características como fertilidade, habilidade materna, precocidade sexual e longevidade, é muito complexa. Em adição, além dos efeitos genéticos e ambientais que sobre ela atuam, ocorrem, em alguns casos, limitações em termos de disponibilidade de informações sobre a vida reprodutiva das matrizes e/ou sobre o desempenho de seus produtos, dificultando a interpretação dos resultados (Rosa, 1999).

Segundo Lôbo et al. (2002), touros com maiores diferenças esperadas na progênie (DEPs) para PAC devem ser utilizados, pois o criador estará selecionando, concomitantemente, para a habilidade materna, fertilidade e precocidade sexual.

Em estudos recentes, Rosa (1999), trabalhando com dados de rebanhos do Programa de Melhoramento Genético da Raça Nelore (PMGRN) no Brasil, observou média de $144 \mathrm{~kg}$ para PAC, constatando efeito significativo $(\mathrm{P}<0,0001)$ de fazenda e ano de nascimento da vaca sobre esta característica. Schwengber (2001), também trabalhando com informações geradas em rebanhos do PMGRN, obtiveram média para PAC de $130 \mathrm{~kg}$ de bezerros desmamados por vaca ao ano, tendo como efeitos significativos $(\mathrm{P}<0,0001)$ pai da vaca, rebanho e ano de nascimento da vaca. Os últimos autores estimaram coeficiente de herdabilidade de 0,15 para a PAC.

Considerando que é de grande importância o conhecimento dos aspectos reprodutivos nas diferentes regiões do país, este trabalho foi conduzido com os objetivos de estimar os componentes de variância e determinar a herdabilidade da PAC em rebanhos das regiões Norte e Nordeste do Brasil, recém incorporados ao Programa de Melhoramento Genético da Raça Nelore, a fim de averiguar a viabilidade da seleção de reprodutores com base nessa característica e, assim, incrementar a produtividade dos rebanhos das citadas regiões.

\section{Material e Métodos}

Os registros utilizados neste estudo foram obtidos de 2.816 fêmeas da raça Nelore, sendo 831 da raça Nelore Mocho e 1.985 da raça Nelore Padrão, nascidas no período de 1982 a 1998, em dez rebanhos localizados nos estados do Pará, Tocantins, Maranhão e Bahia, integrantes do Programa de Melhoramento Genético da Raça Nelore (PMGRN). Os dois primeiros Estados situam-se na região Norte do Brasil, onde predomina o clima equatorial úmido, sem diferenças acentuadas de temperatura ao longo do ano; os outros dois (Maranhão e Bahia) pertencem à região Nordeste, com clima tropical quente e úmido, com inverno seco e verão chuvoso, apresentando temperaturas elevadas durante $o$ ano.

R. Bras. Zootec., v.34, n.1, p.54-59, 2005 
Os rebanhos avaliados foram incluídos recentemente no PMGRN e, desta forma, os dados utilizados englobam também aqueles anteriores à incorporação das técnicas de manejo sugeridas pelo Programa. Os animais foram criados em pastagem, sendo os touros manejados junto com as fêmeas durante todo o ano, não sendo adotada estação de monta como prática de manejo reprodutivo.

A produtividade acumulada (PAC) foi obtida pela aplicação da expressão citada por Lôbo et al. (2000), apresentada a seguir:

$$
P A C=\frac{P_{d} \times n_{p} \times C_{a}}{I V P_{n}-C_{i}}
$$

em que: $\mathrm{PAC}=$ produtividade acumulada $(\mathrm{em} \mathrm{kg}$ de bezerros desmamados por ano); $\mathrm{P}_{\mathrm{d}}=$ média de peso dos bezerros ao desmame (em $\mathrm{kg}) ; \mathrm{n}_{\mathrm{p}}=$ número total de bezerros produzidos pela vaca (bezerros nascidos); $\mathrm{C}_{\mathrm{a}}=$ constante igual a 365 dias que permite expressar a fertilidade em base anual; $\mathrm{IVP}_{\mathrm{n}}=$ idade da vaca ao último parto (em dias); $\mathrm{C}_{\mathrm{i}}=$ constante, igual a 550 dias, aproximadamente 18 meses, utilizada com base na expectativa de o primeiro parto ocorrer ao redor de 30 meses de idade, como meta do PMGRN.

Para determinação da PAC, foram utilizadas vacas com idade mínima e máxima ao último parto de 2.030 dias e 5.116 dias, respectivamente, e com número de bezerros variando de 1 a 11 . Na análise dos dados, foram utilizadas somente as fêmeas que possuíam todos os registros necessários para a determinação da PAC. Foram utilizados 12.193 pesos de bezerros ao desmame, sendo este peso padronizado para a idade de 240 dias.

O procedimento MIXED do pacote estatístico SAS (SAS, 1999) foi utilizado para testar diversos modelos com os efeitos fixos de fazenda, mês ou estação de nascimento da vaca, ano de nascimento da vaca, raça da matriz, e suas interações, além de grupo contemporâneo, composto por fazenda, estação e ano de nascimento da vaca. O modelo selecionado incluiu grupo contemporâneo e raça da matriz como efeitos fixos.

A estação de nascimento da vaca compreendeu os meses de janeiro a março (estação um), abril a junho (estação dois), julho a setembro (estação três) e outubro a dezembro (estação quatro).

R. Bras. Zootec., v.34, n.1, p.54-59, 2005
As análises estatísticas para obtenção dos parâmetros genéticos e fenotípicos foram realizadas pelo método da Máxima Verossimilhança Restrita Livre de Derivada, pela aplicação do software MTDFREML (Boldman et al., 1995), obtidos por modelo animal, de acordo com o modelo misto descrito a seguir:

$$
\mathrm{Y}=\mathrm{X} \beta+\mathrm{Z}_{1} \mathrm{a}+\mathrm{e}
$$

em que: $\mathbf{Y}=$ vetor de observações da característica; $\mathbf{X}=$ matriz de incidência dos efeitos fixos; $\boldsymbol{\beta}=$ vetor dos efeitos fixos; $\mathbf{Z}_{\mathbf{1}}=$ matriz de incidência do efeito genético direto de cada animal; $\mathbf{a}=$ vetor de efeitos genéticos diretos aleatórios; $\mathbf{e}=$ vetor de efeitos residuais aleatórios.

As seguintes pressuposições foram assumidas: $\mathrm{y}$, a e e possuem distribuição normal multivariada; $\mathrm{V}(\mathrm{a})=\mathrm{A} \sigma_{\mathrm{a}}^{2} ; \mathrm{V}(\mathrm{e})=\mathrm{R}=\mathrm{I}^{2}{ }_{\mathrm{e}} ; \operatorname{Cov}(\mathrm{a}, \mathrm{e})=0$; em que $\mathrm{A}=$ matriz de relações genético-aditivas entre os indivíduos, de ordem igual ao número de indivíduos; $\sigma^{2}{ }_{a}=$ variância genética aditiva; $\mathrm{I}=$ matriz identidade de ordem igual ao número de observações e $\sigma^{2}{ }_{\mathrm{e}}=$ variância residual.

\section{Resultados e Discussão}

A média da produtividade acumulada (PAC) e respectivo desvio-padrão para as informações utilizadas neste estudo foram $96,74 \mathrm{~kg} \pm 46,70 \mathrm{~kg}$ de bezerros desmamados por vaca ao ano. A média obtida foi inferior à relatada por Rosa (1999), de 144kg, e por Schwengber (2001), de $130 \mathrm{~kg}$ de bezerros desmamados por vaca ao ano, ambos em rebanhos participantes do PMGRN. Esta diferença observada deve-se, provavelmente, à superioridade no manejo dos rebanhos utilizados pelos autores citados, que trabalharam com rebanhos localizados em diversas regiões do Brasil, incluindo aquelas em que as peculiaridades climáticas favorecem a pecuária e, como conseqüência, diluem os baixos resultados encontrados em rebanhos das regiões Norte e Nordeste do Brasil, como é o caso desta pesquisa.

O coeficiente de variação $(48,27 \%)$ foi superior ao encontrado por Rosa (1999), de 26,5\%, provavelmente, em decorrência da maior variabilidade dos dados entre vacas quanto à idade ao último parto e ao número e peso dos bezerros desmamados, em decorrência da própria estrutura e manejo dos rebanhos.

Na formação dos grupos contemporâneos, foram incluídos os efeitos de rebanho e estação e ano de nascimento da vaca. Diferenças entre grupos con- 
temporâneos decorreram, provavelmente, de alterações climáticas entre as regiões onde os rebanhos se localizam, visto que, entre os dez avaliados, seis situam-se no estado da Bahia, cuja pluviosidade característica é inferior à dos demais estados, influenciando a disponibilidade de forragem aos animais, criados em pastagem. Outros fatores que podem concorrer para diferenças entre os grupos contemporâneos são as práticas de manejo nutricional (suplementação ou não em períodos de seca prolongada), reprodutivo e sanitário adotadas e, também, os diferentes métodos de seleção escolhidos pelos criadores entre propriedades, que contribuem para o aumento da diversidade genética dos animais.

É importante ressaltar que os rebanhos avaliados foram recém incorporados ao PMGRN, sendo, portanto, a maioria das observações utilizadas neste trabalho adotadas anteriormente às recomendações do Programa quanto às práticas de manejo. Dessa forma, acredita-se que a simples implementação de estação de monta em época e duração adequadas, realizada após rigoroso controle dos aspectos reprodutivos dos machos e fêmeas, bem como o acúmulo de informações com o decorrer do tempo, possa vir a incrementar a PAC nestes rebanhos e em outros que venham a ingressar no PMGRN.

Neste contexto, a PAC deve ter sua importância ressaltada como critério de seleção de reprodutores, por contemplar não apenas a taxa de prenhez ou desmama da vaca ao longo de sua vida útil, mas também a produção periódica de progênie mais pesada o que acarreta maior retorno econômico por vaca.

A raça da matriz teve efeito significativo $(\mathrm{P}<0,01)$ sobre a PAC. Fêmeas Nelore Padrão foram superiores às fêmeas Mocha, em razão, provavelmente, da menor idade ao primeiro parto ou do menor intervalo de partos, ou ainda, de maior habilidade materna das primeiras, ocasionando a desmama de animais mais pesados.

Os componentes de variância e a estimativa de herdabilidade para PAC são apresentados na Tabela 1. A análise dos valores dos componentes de variância ressalta a participação do componente de ambiente, que representa cerca de $89 \%$ da variância fenotípica. A estacionalidade da produção de pastagem pode ser considerada uma das prováveis causas do retardo da idade à primeira cria e do prolongamento do intervalo de partos, características diretamente relacionadas à eficiência reprodutiva e a PAC.
Tabela 1 - Estimativas dos componentes de variância e da herdabilidade para produtividade acumulada (PAC) em rebanhos Nelore criados no Norte e Nordeste do Brasil

Table 1 - Components of variance and heritability for accumulated productivity (ACP) in Nelore herds raised in the North and Northeast of Brazil

\begin{tabular}{lcccc}
\hline & $\sigma^{2}{ }_{\mathrm{a}}$ & $\sigma_{\mathrm{e}}^{2}$ & $\sigma_{\mathrm{f}}^{2}$ & $\mathrm{~h}^{2}$ \\
\hline $\operatorname{PAC}(A C P)$ & 95,16 & 748,01 & 843,17 & $0,11 \pm 0,06$
\end{tabular}

$\sigma^{2}{ }_{a}=$ variância genética aditiva (genetic additive variance); $\sigma^{22}{ }_{\mathrm{e}}=$ variância de ambiente (environmental variance); $\sigma^{22}{ }_{\mathrm{f}}=$ variância fenotípica (phenotypic variance); $\mathrm{h}^{2}=$ herdabilidade (heritability).

O coeficiente de herdabilidade obtido $(0,11 \pm 0,06)$ foi de magnitude semelhante ao estimado por Schwengber et al. (2001), de 0,15 , porém inferior ao encontrado por Rosa (1999), de 0,19. O baixo valor de herdabilidade deve-se, provavelmente, ao fato desta variável ser composta por outras características, de caráter reprodutivo e, portanto, de herdabilidade inferior às características de produção.

Infere-se, portanto, que a baixa herdabilidade estimada corrobora a citação de Notter (1995) de que a herdabilidade dos índices tende a ser controlada pela característica mais variável e menos herdável, apesar da presença, no mesmo índice, de características de maior herdabilidade. Assim, observando-se que a PAC inclui características como precocidade e periodicidade reprodutiva, ambas comumente citadas na literatura como de reduzida herdabilidade $(0,07-$ Biffani, 1999; 0,15 - Garnero et al., 1999; 0,10 Schwengber et al., 2002), é esperado que o valor da herdabilidade para PAC seja mesmo baixo. Deve-se ressaltar que, entre as características reprodutivas, a idade ao primeiro parto pode apresentar herdabilidade de moderada à alta magnitude $(0,46$ - Pereira et al., 1991; 0,28 - Mercadante et al., 2000; 0,27 - De los Reyes-Borjas et al., 2002), sobretudo quando as fêmeas são desafiadas já aos 12 meses de idade (0,57 - Eler et al., 2002).

Neste trabalho, o valor estimado para herdabilidade da PAC está próximo aos obtidos para características reprodutivas, como intervalo de partos (0,07 - Martins Filho et al., 1995; 0,08 - Biffani, 1999; 0,10 - Gresller et al., 2000) e fertilidade real (0,13 - Mercadante et al., 1996), sendo, porém, inferior ao freqüentemente encontrado para peso do bezerro ao desmame (0,29 - Martins et al., 1996; 0,28 - Ferraz Filho et al., 1998), que reflete, além da 
capacidade de crescimento do bezerro, a produção de leite da fêmea e sua capacidade de cuidar da cria.

A baixa herdabilidade estimada denota que o valor obtido para PAC poderia ser mais rapidamente incrementado ao serem utilizadas práticas de manejo que melhorassem as condições nutricionais, sanitárias e reprodutivas dos animais. No entanto, deve-se ressaltarque há variabilidade genética aditiva suficiente para que esta característica seja incluída como critério de seleção em programas de melhoramento genético, o que ocasionaria maior produtividade das fêmeas do rebanho a partir da escolha de reprodutores avaliados e considerados superiores para PAC. Dessa forma, o criador, ao utilizar estes reprodutores superiores, estaria melhorando habilidade materna, fertilidade e precocidade sexual em seu rebanho, simultaneamente.

\section{Conclusões}

A baixa média de produtividade acumulada, obtida para os rebanhos das regiões Norte e Nordeste avaliados, ressalta a necessidade de melhor manejo geral, sobretudo quanto à nutrição e reprodução nestas propriedades.

A herdabilidade estimada para produtividade acumulada, embora de baixa magnitude, sugere a existência de variabilidade genética suficiente para inclusão dessa característica em programas de melhoramento genético, com a ressalva de que seu progresso depende, em maior parte, de práticas de manejo mais eficientes.

Considerando que a produtividade acumulada é um índice que incorpora características fundamentais à seleção de fêmeas, a herdabilidade estimada indica possibilidade de obtenção de fêmeas mais produtivas avaliadas por sua periodicidade reprodutiva e pelo peso dos seus bezerros ao desmame, o que, em conseqüência, proporcionaria maior produtividade potencial dos rebanhos.

\section{Literatura Citada}

BIFFANI, S. Caratteristiche riproduttive di femmine di razza Chianina e Nelore allevate nel Nord Este del Brasile. Firenze: Università degli Studi di Firenze, 1999. 104p. Tese (Doutorado em Scienze Zootecniche) - Università degli Studi di Firenze, 1999.

BOLDMAN, K.G.; KRIESE, L.A.; Van VLECK, L.D. et al. A manual for use of MTDFREML - a set of programs to obtain estimates of variances and covariances (DRAFT). Lincoln: USDA-ARS, 1995. 120p.

R. Bras. Zootec., v.34, n.1, p.54-59, 2005
CAMPELlO, C.C.; MARTINS FILHO, R.; LÔBO, R.N.B. et al. Intervalo de partos e fertilidade real em vacas Nelore no estado do Maranhão. Revista Brasileira de Zootecnia, v.28, n.3, p.474-479, 1999.

DE LOS REYES-BORJAS, A.; MAGNABOSCO, C.U.; LÔBO, R.B. et al. Estimativas de (co)variância e parâmetros genéticos para dias ao parto e características relacionadas em fêmeas Nelore. In: REUNIÃO ANUAL DA SOCIEDADE BRASILEIRA DE ZOOTECNIA, 39., 2002, Recife. Anais... Recife: Sociedade Brasileira de Zootecnia, 2002. 1 CD.

DINKEL, C.A.; BROWN, M.A. An evaluation of the ratio of calf weight to cow weight as an indicator of cow efficiency. Journal of Animal Science, v.46, n.3, p.614-617, 1978.

ELER, J.P., SILVA, J.A. II V.; FERRAZ, J.B.S. et al. Genetic evaluation of the probability of pregnancy at 14 month for Nellore heifers. Journal of Animal Science, v.80, n.4, p.951-954, 2002.

EUCLIDES FILHO, K.; RESTLE, J.; OLSON, T.A. et al. Medidas de eficiência reprodutiva na produção de terneiros a partir de vacas de tamanho e habilidade leiteira diferentes. In: REUNIÃO DA SOCIEDADE BRASILEIRA DE ZOOTECNIA, 21., 1984, Belo Horizonte. Anais... Belo Horizonte: Sociedade Brasileira de Zootecnia, 1984. p.138.

FERRAZ FILHO, P.B.; BIANCHINI SOBRINHO, E.; SILVA, L.O.C. et al. Estimativas de parâmetros genéticos e fenotípicos para pesos na raça Nelore mocha em três regiões Brasileiras. In: REUNIAO ANUAL DA SOCIEDADE BRASILEIRA DE ZOOTECNIA, 35., 1998, Botucatu. Anais... Botucatu: Sociedade Brasileira de Zootecnia, 1998. p.517-519.

GARNERO, A.V.; LÔBO, R.B., BEZERRA, L.A.F. et al. Estimativas de parâmetros genéticos de características reprodutivas na raça Nelore. In: REUNIÃO ANUAL DA SOCIEDADE BRASILEIRA DE ZOOTECNIA, 36., 1999, Porto Alegre. Anais... Porto Alegre: Sociedade Brasileira de Zootecnia, 1999. 1 CD.

GRESSLER, S.L.; BERGMANN, J.A.G.; PEREIRA, C.S. et al. Estudo das associações genéticas entre perímetro escrotal e características reprodutivas de fêmeas Nelore. Revista Brasileira de Zootecnia, v.29, n.2, p.427-437, 2000.

GONÇALVES, J.N.S.; SCARPATI, M.T.V.; NARDON, R.F. et al. Avaliação da fertilidade real e da capacidade mais provável de fertilidade real de matrizes de um rebanho da raça Nelore. In: REUNIÃO ANUAL DA SOCIEDADE BRASILEIRA DE ZOOTECNIA, 33., 1996, Fortaleza. Anais... Fortaleza: Sociedade Brasileira de Zootecnia, 1996. p.368.

KIRKPATRICK, B.W.; DINKEL, C.A.; RUTLEDGE, J.J. et al. Prediction equations of beef cow efficiency. Journal of Animal Science, v.60, n.4, p.964-969, 1985.

LÔBO, R.B.; BEZERRA, L.A.; OLIVEIRA, H.N. et al. Avaliação genética de animais jovens, touros e matrizes. Ribeirão Preto: Universidade de São Paulo/GEMAC, 2000. 90p.

LÔBO, R.B. Programa de melhoramento genético da raça Nelore. 2.ed. Ribeirão Preto: FINEP, 1994. 100p.

LÔBO, R.B.; BEZERRA, L.A.F.; OLIVEIRA, H.N. et al. Avaliação genética de animais jovens, touros e matrizes. Ribeirão Preto: Universidade de São Paulo/GEMAC, 2002.76p.

MARTINS, G.A.; MARTINS FILHO, R.; LÔBO, R.N.B. Fatores genéticos e de ambiente que influenciam o peso à desmama em bovinos da raça Nelore. In: REUNIÃO ANUAL DA SOCIEDADE BRASILEIRA DE ZOOTECNIA, 33., 1996, Fortaleza. Anais... Fortaleza: Sociedade Brasileira de Zootecnia, 1996. v.1. p.181-183. 
MARTINS FILHO, R.; LÔBO, R.B.; LIMA, F.A.M. Intervalo entre partos de vacas Nelore no estado de São Paulo. In: REUNIÃO ANUAL DA SOCIEDADE BRASILEIRA DE ZOOTECNIA, 32., 1995, Brasília. Anais... Brasília: Sociedade Brasileira de Zootecnia, 1995. p.422.

MERCADANTE, M.E.Z.; LÔBO, R.B.; BORJAS, A.L.R. et al. Estudo genético-quantitativo de características de reprodução e produção em fêmeas da raça Nelore. In: REUNIÃO ANUAL DA SOCIEDADE BRASILEIRA DE ZOOTECNIA, 33., 1996, Fortaleza. Anais... Fortaleza: Sociedade Brasileira de Zootecnia, 1996. v.1. p.155-157.

MERCADANTE, M.E.Z.; LÔBO, R.B.; OLIVEIRA, H.N. Estimativas de (co)variâncias entre características de reprodução e de crescimento em fêmeas de um rebanho Nelore. Revista Brasileira de Zootecnia, v.29, n.4, p.997-1004, 2000.

NOTTER, D.R. Maximizing fertility in animal breeding programs. In: CONGRESSO BRASILEIRO DE REPRODUÇÃO ANIMAL, 11., 1995, Belo Horizonte. Anais... Belo Horizonte: Colégio Brasileiro de Reprodução Animal, 1995. p.36.

PEREIRA, J.C.C.; AYALA, J.M.N., OLIVEIRA, A.N. Efeitos genéticos e não genéticos sobre a idade ao primeiro parto e o intervalo entre partos de duas populações da raça Nelore. Arquivo Brasileiro de Medicina Veterinária e Zootecnia, v.42, n.1, p.93-102, 1991.

RAHNEFELD, G.W.; WEISS, G.M.; WARD, D. A comparison of methods to evaluate beef cow productivity. Canadian Journal Animal, v.73, n.4, p.971-975, 1993.
ROSA, A.N. Variabilidade fenotípica e genética do peso adulto e da produtividade acumulada de matrizes em rebanhos de seleção da raça Nelore no Brasil. Ribeirão Preto: Universidade de São Paulo, 1999. 114p. Tese (Doutorado em Ciências) - Universidade de São Paulo.

STATISTICAL ANALYSES SYSTEM - SAS. SAS/STAT ${ }^{\text {TM. }}$ SAS user's guide for windows environment. version 6 . 11.ed. Cary: 1999.

SCHWENGBER, E.B. Produtividade acumulada e peso adulto como características alternativas na seleção de fêmeas da raça Nelore. Ribeirão Preto: Universidade de São Paulo, 2001. 103p. Tese (Doutorado em Ciências) - Universidade de São Paulo, 2001.

SCHWENGBER, E.B.; BEZERRA, L.A.F.; LÔBO, R.B. Produtividade acumulada como critério de seleção em fêmeas da raça Nelore. Ciência Rural, v.31, n.3, p.483-486, 2001.

SCHWENGBER, E.B.; LÔBO, R.B.; BEZERRA, L.A.F. Parâmetros genéticos da idade à primeira cria, intervalo de partos e período de gestação na raça Nelore. In: REUNIÃO ANUAL DA SOCIEDADE BRASILEIRA DE ZOOTECNIA, 39., 2002, Recife. Anais... Viçosa, MG: Sociedade Brasileira de Zootecnia, 2002. 1 CD.

Recebido em: 23/05/03

Aceito em: $25 / 10 / 04$ 